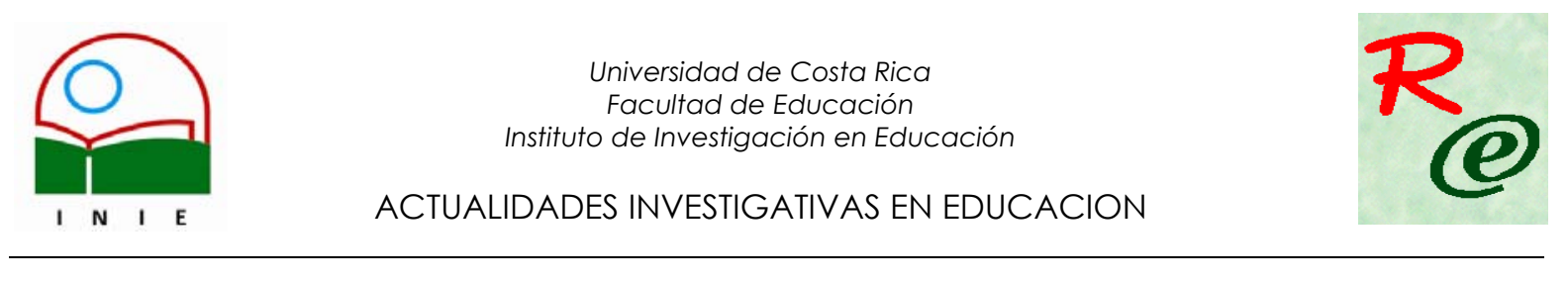

\title{
LA ENSEÑANZA DEL INGLÉS EN COSTA RICA Y LA DESTREZA AUDITIVA EN EL AULA DESDE UNA PERSPECTIVA HISTÓRICA
}

\author{
Patricia Córdoba Cubillo ${ }^{1}$ \\ Rossina Coto Keith ${ }^{2}$ \\ Marlene Ramírez Salas ${ }^{3}$
}

\begin{abstract}
Resumen: Este artículo es parte de una serie de escritos producto de un proyecto de investigación sobre la enseñanza y el aprendizaje de la destreza auditiva en la clase de inglés en colegios públicos de Costa Rica. El mismo pretende proveer una reseña histórica sobre la enseñanza del inglés en Costa Rica y la destreza auditiva en una segunda lengua en general. El artículo está dividido en dos grandes secciones. La primera sección se concentra en la enseñanza del inglés en Costa Rica, desde alrededor de 1850 hasta nuestros días. La segunda parte trata sobre la enseñanza de la destreza auditiva en el aula, a la luz de los diferentes métodos que se han utilizado para la enseñanza de una segunda lengua o lengua extranjera.
\end{abstract}

Palabras claves: HISTORIA/ ENSEÑANZA DEL INGLÉS/ DESTREZA AUDITIVA/ AULA/ MÉTODOS/

\begin{abstract}
This article is the first in a series of pieces that are the result of a research project about the teaching and learning of the listening skill in the English class in public high schools in Costa Rica. It intends to give a historical overview of the teaching of English in Costa Rica and the teaching of the listening skill in general. The article is divided in two sections. The first part centers on the teaching of English in Costa Rica from around 1850 up to the present. The second part deals with the teaching of the skill through history in light of the different methods that have been used to instruct in a second or foreign language.
\end{abstract}

Key words: HISTORY/ ENGLISH TEACHING/ LISTENING SKILL/ CLASSROOM/ METHODS/

\begin{abstract}
${ }^{1}$ Magíster en la Enseñanza del Inglés como Lengua Extranjera; Licenciatura en Lingüística, Bachiller en Inglés, Profesora de Enseñanza Media en el ramo de Inglés, todos los títulos de la Universidad de Costa Rica. Profesora de la Escuela de Lenguas Modernas de la Universidad de Costa Rica. Correo electrónico: pcordoba@racsa.co.cr

${ }^{2}$ Maestría en la Enseñanza del Inglés como Segunda Lengua de la University of Northern lowa. Bachiller en Inglés de la Universidad de Costa Rica. Profesora de la Escuela de Lenguas Modernas de la misma universidad. Correo electrónico: rossinacoto@yahoo.com

${ }^{3}$ Master en la Enseñanza del Inglés como Lengua Extranjera de Southern Illinois University. Bachiller en Inglés de la Universidad de Costa Rica. Labora para la Universidad de Costa Rica desde 1993 en la Escuela de Lenguas Modernas impartiendo cursos a nivel de Bachillerato, Licenciatura y Maestría. Correo electrónico:
\end{abstract} marami@racsa.co.cr

Artículo recibido: 6 de julio, 2005

Aprobado: 31 de octubre, 2005 
A través de la historia, desde la división de los lenguajes en la Torre de Babel, uno de los anhelos más grandes de gran parte de la humanidad ha sido poder expresarse no sólo en su propia lengua, sino en las lenguas que otros grupos o culturas utilizan para poder comunicarse con su grupo social. En Costa Rica, este deseo se expresó con la introducción, primeramente, del latín en la educación de la incipiente sociedad costarricense. Luego, con el correr de los años $y$, debido a la influencia de otras sociedades y de las telecomunicaciones, se reemplazó la enseñanza del latín por la enseñanza de otros idiomas, en especial el francés y el inglés. Este último idioma, por diversas razones geográficas, económicas, políticas, sociales y culturales, ha contado con más influencia en nuestro país, sin demérito de los otros idiomas que se enseñan en Costa Rica. Es por esta razón que el estudio de la enseñanza del inglés en Costa Rica y el aporte de ideas para su mejoramiento se hacen fundamentales en nuestros días. Dentro de esta tendencia se destaca la escasa y, en muchos casos, nula importancia que se ha dado a la instrucción de la destreza auditiva en el aula de secundaria.

Como parte de un proyecto de investigación en curso $^{4} \mathrm{y}$, como un interés en proporcionar ideas y materiales, y así colaborar con el proceso educativo costarricense, en especial la enseñanza del inglés en Costa Rica, este escrito es parte de una serie de artículos sobre el estado actual de la enseñanza de la destreza auditiva en la clase de inglés en secundaria y el aporte de soluciones relacionadas con el tema en cuestión. La intención de las autoras es que los estudiantes de secundaria incrementen su nivel en el idioma inglés, a través de diversas actividades de escucha congruentes con el Plan de Estudios del Ministerio de Educación Pública para estudiantes de sétimo año, lo que ofrecería mejores oportunidades educativas, sociales y laborales para los miembros de la sociedad costarricense. Pero para lograr comprender mejor esta realidad y lograr así proporcionar soluciones congruentes con nuestra historia y nuestro contexto social y cultural actual, es fundamental presentar un repaso histórico sobre la enseñanza del inglés en Costa Rica y sobre la destreza auditiva en sí.

Para alcanzar este propósito, este artículo cubre dos grandes áreas. La primera parte ofrece una reseña histórica sobre la enseñanza del inglés en Costa Rica desde el siglo XIX. La segunda parte realiza una labor similar en cuanto a la enseñanza de la destreza auditiva

4 Este proyecto de investigación se encuentra inscrito por las autoras en la Vicerrectoría de Investigación de la Universidad de Costa Rica bajo el título "La destreza auditiva en inglés en seis colegios públicos académicos del Área Metropolitana: Importancia, estado de la cuestión, problemas y soluciones" y el código: 023-A4-155 
en el aula, en relación con los diferentes métodos que se han utilizado a través de la historia para la instrucción en idiomas extranjeros.

\section{La enseñanza del inglés en Costa Rica ${ }^{5}$}

En nuestro país la enseñanza de idiomas, especialmente del inglés y del francés, se inició a mediados del siglo XIX. Como lo afirma González (1978, p. 345), "la enseñanza de idiomas extranjeros despertó ya el interés en los primeros años de la República.". En relación con la enseñanza del inglés en nuestro país, la primera referencia que podemos encontrar data del año 1825, cuando el Poder Ejecutivo decretó el reglamento interno de la Casa de Enseñanza Santo Tomás, iniciándose así el estudio de lenguas en forma institucional. Según González (1978), el plan de estudios de la Casa de Enseñanza ofrecía clases de latín, francés e inglés. En esta época, la metodología se basaba en la memorización de lecciones por parte del estudiante, las cuales luego eran examinadas en forma oral por el maestro. El tiempo dedicado al estudio del idioma inglés era de tres horas diarias (pp. 203- 204).

A partir de 1854, surgieron las primeras ofertas de profesores extranjeros para impartir clases privadas de inglés y otros idiomas (González, 1978, p. 345). Aunque no se conoce con certeza el método de enseñanza utilizado en estos años, es de suponer que se basaba en la Traducción Gramatical, método de gran auge en la enseñanza de las lenguas clásicas que fue adoptado para la enseñanza de las lenguas modernas. De esta forma, la enseñanza del idioma inglés formó parte de nuestro sistema educativo desde sus inicios. Con la creación de los colegios primario - secundarios, precursores de los colegios de segunda enseñanza, las lecciones de inglés se vuelven parte de los planes de estudio (Quesada, 1993, p. 22). Algunos ejemplos de esta innovación lo constituyen: el Colegio de Humanidades de Jesús, (1859); el Colegio de Humanidades Páez, (1861); el Colegio de Alajuela, (1866); el Colegio de Heredia, (1870); el Colegio Santo Tomás, (1873); el Colegio Josefino, (1877) y el Colegio de Señoritas, (1888) (González, 1978, pp. 369-377). Los planes de estudios de estos colegios incluían tres clases de inglés a la semana, las cuales eran impartidas por profesores nacionales y extranjeros. El método de Traducción Gramatical se

\footnotetext{
${ }^{5}$ La mayor parte de esta reseña bibliográfica fue tomada directamente de una entrevista personal en setiembre del 2004 realizada a las licenciadas Ana Lorena Araya U. y Mayela Morera R., quienes elaboraron en 1997 una Guía de Actividades para la Enseñanza del Inglés como Lengua Extranjera en el Primer Ciclo de la Enseñanza General Básica, como Proyecto de Graduación para el grado de Licenciatura en la UNED.
} 
utilizó por varios años. En esta época varios de estos colegios utilizaban el texto de inglés de Ollendorff y Robertsons (González, 1978, pp. 374-375, 445-455).

En 1887 se establecieron los colegios secundarios, los cuales también incluyeron el inglés dentro de sus planes de estudios. Según González (1976), se adoptó el Método Directo Alemán, con el estudio de las obras del Dr. Rodolfo Lenz y Schnitzler (1976, p. 76). Este método establece que el aprendizaje de una segunda lengua debe ser igual al de la primera, espontáneo, con mucha actividad oral, sin usar la traducción y con poco o ningún análisis de reglas gramaticales (Brown, 2000, p. 45). Estos planes se mantuvieron vigentes por varias décadas, aunque sin certeza de cuánto tiempo fueron utilizados, ya que no se encuentra información documental sobre el tema entre los años 1887 y 1934. En 1935, tras un análisis de la situación educativa de nuestro país $\mathrm{y}$, teniendo en mente la necesidad inherente de renovación de la educación, se dieron varios cambios en el sistema educativo costarricense, los cuales transformaron la enseñanza del inglés. Luego de haber analizado los planes de estudio y con una visión modernizadora, la Junta de Directores de Segunda Enseñanza, único organismo coordinador de la educación costarricense para entonces, aprobó un plan de cinco años que entró en vigencia en 1936. En dicho plan se distinguían tres tipos de áreas: filológico-histórico; científico-matemático y técnico-artístico. Dentro de la primera categoría, se incluía el inglés como parte del bloque de materias. De este modo, se impartían cuatro lecciones en el primero y segundo años y tres en tercero, cuarto y quinto años (Monge y Rivas, 1978, pp. 92-93). En relación con este plan, la licenciada Elsa Orozco acotó en su momento que los libros que se utilizaban en 1942 no tenían ninguna relación con el programa establecido en 1936 (Cabrera, 1986, p. 2).

Hasta ese momento, las personas que impartían las clases de inglés en secundaria eran extranjeros (los cuales enseñaban su lengua materna) o nacionales (quienes aprendieron el idioma mientras estudiaban en el exterior) que carecían de la preparación pedagógica necesaria para ejercer en el campo educativo. Probablemente, y debido a esta situación, la Universidad de Costa Rica ofreció por primera vez en 1954 cursos de capacitación para los profesores de inglés. En dichos cursos la metodología que se impartía para que el docente utilizara en sus lecciones era el método Audiolingual (Cabrera, 1986, p.4).

Sin embargo, no es hasta 1957 cuando se profesionaliza la enseñanza del inglés en Costa Rica, con la apertura de la carrera de inglés en la Facultad de Educación de la Universidad de Costa Rica. El plan de estudios de esta carrera tenía una duración de cuatro años e incluía lengua, literatura, metodología y práctica docente. El enfoque utilizado era el 
Método Audiolingual. Se utilizaban textos como: la serie English Pattern Practices, Lessons in Vocabulary and English Sentence Patterns ambos de Robert Lado y Charles Fries; Reading in English de Dorothy Danielson y Rebeca Hayden; Literature of America y Literature of England de Mildred Foster, y Basic Speech de Jon Erickson y Paul H. Boase.

Debido a estos cambios, en los planes de estudio de secundaria se establecieron tres lecciones de idioma (inglés o francés) en el primer ciclo (lo que es actualmente el tercer ciclo) y tres o cuatro en el segundo ciclo de secundaria (lo que constituye actualmente la educación diversificada) (Monge y Rivas, 1978, pp.195-196). Los profesores de lengua extranjera y el Ministerio de Educación implementan el Método Audiolingual con el fin de desarrollar las cuatro destrezas básicas: comprensión oral, producción oral, comprensión de lectura y producción escrita. Para ello se aplica la repetición de patrones, memorización de diálogos y mímica. El libro de texto que se usó a partir de este momento fue American English Series (libros 1, 2 y 3 ) de Charles Fries, el cual estuvo en vigencia hasta alrededor del año 1972 (Cabrera, 1986, p. 6).

En 1966, se editan los planes de estudio para el tercer ciclo, bajo la coordinación de la señora Nidia Herrera Rodríguez, Asesora Nacional de Inglés. En 1972, se instaura el Plan de Desarrollo Educativo para reexaminar y replantear integralmente el quehacer educativo de nuestro país (MEP, 1972). Con base en el plan existente y, en el año antes citado, se elabora un nuevo plan de estudios para la enseñanza del inglés. Este comprende una especie de guía que contiene objetivos, actividades y formas de evaluación para que el maestro lleve a cabo sus lecciones. Dicho plan no se apega a un texto específico, sino más bien menciona en su bibliografía varios textos que podrían ser utilizados por el docente, entre ellos, Lado English Series (libros 1, 2 y 3) de Robert Lado en el área académica, Modern American English de Robert J. Dixon en la rama técnica vocacional, y New Horizons in English de Lars Mellgren, en los colegios nocturnos. También en algunos colegios se usó como libro de apoyo el Let's Learn English de Audrey Wright (MEP, 1972). La metodología de estos libros se basa principalmente en la forma típica en que se aprende la lengua materna (comprensión oral, producción oral, lectura y escritura). En 1974, se hizo una nueva edición de este plan para el tercer ciclo, lo que hace pensar que funcionó en forma exitosa hasta entonces. El número de lecciones semanales y la metodología se mantienen sin cambios, pero se presenta un mayor énfasis en la pronunciación.

Alrededor de 1978 y hasta 1990, el Ministerio de Educación Pública (MEP) elimina el plan de estudios y, en su lugar, prepara y envía a los docentes una guía muy simple en la que se indican los libros que se deben usar y las unidades que se deben cubrir en cada 
nivel. Se continúan utilizando los libros mencionados anteriormente excepto por Let's Learn English de Wright. Además se indica el uso del libro English for a Changing World de Ronald Wardhaugh en los colegios académicos diurnos (Cabrera, 1986, p. 7). Este libro se basa en la misma metodología de los textos usados anteriormente, pero además, fomenta el uso de situaciones comunicativas en la clase. Dicha guía no establece objetivos, actividades, ni formas de evaluación, sino que únicamente muestra las unidades que el docente debe completar.

En 1988, con el restablecimiento del examen de bachillerato, el número de lecciones semanales aumenta a cinco en la educación diversificada, lo que trae, como consecuencia, ciertos cambios en la metodología, al enfatizarse la comprensión de lectura y el análisis de textos en inglés tomados de diversas fuentes.

En 1990 se da un cambio radical, pues se elaboran otros planes que establecen nuevos objetivos, nuevas situaciones de aprendizaje y nuevas formas de evaluación. El enfoque se acerca más al Método Comunicativo y, es así cómo, la parte oral toma gran importancia dentro de los planes establecidos. Durante este período se da la creación de nuevos libros por parte de profesores nacionales. Con la asesoría del Proyecto LEARN, auspiciado por el gobierno de Inglaterra, se asiste a nuestro país con ideas y materiales actualizados para el mejoramiento de la enseñanza del inglés y se crean libros que se recomiendan como material de apoyo en las lecciones de inglés, tales como Have Fun Libros 1, 2 y 3 de Rosa María Bonilla, et al., para el tercer ciclo. En esta misma década, las Magistras Patricia Córdoba y Leonor Cabrera, junto con la Casa Editorial McGraw Hill, elaboran los libros Learning English, como material complementario para la educación diversificada, específicamente prácticas extra para el examen de bachillerato.

Hasta el año 2005, se continúa utilizando para la enseñanza del inglés el Método Comunicativo basado en el plan de estudios de inglés elaborado por la Comisión Asesora Evaluadora para la enseñanza del inglés del MEP. Los profesores tienen flexibilidad de utilizar varios textos, entre ellos Have Fun de Rosa María Bonilla et al, New Interchange de Jack C. Richards, Super Goal de Manuel dos Santos hasta tercer año y Solutions I y II de Madeline Mc Hugh para cuarto y quinto años. Otros profesores se inclinan por elaborar sus propias antologías, lo que es aceptado por el MEP, en el tanto se cumpla con el plan de estudios.

En cuanto a la enseñanza del inglés en primaria, el 18 de julio de 1994, a raíz de un ofrecimiento de la campaña política de José M. Figueres O., se inició por primera vez, y con la supervisión del programa PROLED (Programa de Lenguas Extranjeras para el Desarrollo), 
un programa de inglés en 27 escuelas públicas. Su objetivo es que la mayoría de los niños costarricenses, sobre todo en áreas rurales, turísticas y zonas marginales, tengan acceso al idioma inglés, con el fin de proporcionarles un nivel cultural que les brinde mejores oportunidades profesionales. Poco a poco se van incorporando a este programa de lenguas extranjeras otros idiomas como el francés y el italiano. El 8 de mayo de 1997, se declara el inglés como materia básica en primero y segundo ciclos. Un año después, en 1998, se inicia el plan piloto para incorporar el inglés en la etapa preescolar. Hasta el año 2003, Costa Rica se destaca como el único país centroamericano que ofrece un modelo de enseñanza de un idioma extranjero en la enseñanza primaria. En el 2004, la Oficina de Lenguas Extranjeras (OLE, antiguamente PROLED) bajo la supervisión de la M.Sc. Anabelle Venegas F. cuenta con 1570 escuelas y 1568 docentes en el programa de inglés, para un total del $73.7 \%$ de cobertura nacional, de acuerdo con el Departamento de Estadística del MEP. Según Venegas, este programa cuenta también con asesores en todo el país, quienes guían y coordinan la enseñanza de idiomas en las diversas escuelas. Además, se promueve la enseñanza del inglés con énfasis en las destrezas oral y auditiva, razón por lo cual no se utiliza ningún texto específico. Los maestros siguen un programa, elaborado por OLE, con diferentes temas y objetivos, y ellos mismos se encargan de buscar el material adecuado para cubrir esos temas (Ramírez, 2004).

Por medio de esta reseña histórica, se aprecia cómo el interés por la enseñanza del idioma inglés en Costa Rica se ha incrementado con el tiempo. Se ha atravesado por una serie de etapas desde finales del siglo XIX, cuando el objetivo era que el estudiante tradujera y comprendiera textos escritos en inglés, hasta nuestros días en que el interés principal está en el aprendizaje y uso del idioma inglés para la comunicación oral. Éstos han sido los cambios más significativos en la transformación y modernización de la enseñanza del inglés en nuestro país, lo que constituye un gran paso para el reto que enfrentará la educación costarricense en el nuevo milenio. Asimismo, con el advenimiento de una serie de academias e institutos tanto públicos como privados, que ofrecen lecciones para el aprendizaje del inglés $u$ otra lengua, se ha dado un incremento en las oportunidades de aprendizaje de un segundo idioma, lo que redunda en mejores beneficios y oportunidades para la población costarricense.

\section{La destreza auditiva a través de la historia}

A lo largo de muchos años, se han utilizado diversos métodos de enseñanza para una lengua extranjera. Cada uno de ellos ha contribuido con un enfoque diferente hacia el 
desarrollo de la destreza auditiva. A continuación se presenta una reseña de algunos métodos de enseñanza de lenguas extranjeras en los que la destreza auditiva tuvo un papel preponderante.

A finales del siglo XIX, aparece el Método Directo (Direct Method). Sus principales exponentes fueron los reformistas Gouin, L. Sauveur, F. Franke y M. Berlitz. Se argumenta, en este momento, que se puede enseñar una lengua extranjera sin necesidad de recurrir a la traducción o al uso de la lengua materna. Richards y Rodgers (2001) afirman que "este método enfatiza la instrucción de la lengua extranjera en la lengua meta. De este modo, los estudiantes tienen que usar su destreza auditiva para comprender los diálogos e historias vistos en clase" (p. 13) ${ }^{6 *}$. Como parte de las técnicas utilizadas por los profesores, Freeman menciona el dictado y la utilización de mapas para localizar ciertos lugares siguiendo las instrucciones del profesor (2000, p. 25). Indudablemente, la efectividad de estos ejercicios depende de una buena comprensión auditiva por parte de los estudiantes. La popularidad de este método llega hasta los años 20, cuando las estrategias de conversación se empiezan a considerar poco prácticas y se le dar mayor énfasis a la lectura.

Entre 1940 y 1950 nace el Método Audiolingual basado en las teorías conductivistas de B.F. Skinner y en la lingüística estructural. El principal representante de este enfoque es Charles Fries, para quien el punto de partida de la enseñanza de una lengua extranjera era la gramática, a través de procedimientos mecánicos de repetición y de desarrollos de hábitos. Se enfatiza la memorización y la repetición de diálogos, la correcta pronunciación y el aprendizaje de la gramática por medio de patrones. Celce-Murcia (1991) apunta que "este método forzaba a los estudiantes a escuchar una oración y reproducirla correctamente con sus elementos léxicos, fonológicos y gramaticales de una forma casi perfecta" (p. 28). Debido a esto, la destreza auditiva juega un papel primordial en este método. Se destaca, también, la importancia de las grabadoras, del equipo audiovisual y de los laboratorios como herramientas de enseñanza-aprendizaje. Algunas de las técnicas de clase usadas en este método y mencionadas por Richards y Rodgers (2001), son la repetición escalonada de oraciones y los pares contrastantes, en los cuales los estudiantes escuchan dos palabras que difieren en un solo sonido, por ejemplo fill / feel; day / they en inglés, o pero / perro en español (pp.60-61). A través de estos ejercicios, los estudiantes discriminan sonidos producidos en una misma área de articulación, o en su entorno, pero con características diferentes como sonoridad y alargamiento. El Método Audiolingual le da gran relevancia a la

\footnotetext{
${ }^{6}$ Traducción de las autoras.
} 
destreza auditiva; sin embargo, no tiene mucho éxito en promover una enseñanza más espontánea en donde los estudiantes puedan utilizar situaciones de la vida real de forma creativa.

A mediados de los años 60, surge el Método de Repuesta Física Total (TPR) desarrollado por J. Asher. Este método pretende enseñar el idioma meta por medio de órdenes y actividad física en medio de un ambiente agradable y sin tensiones. Se requiere, también, de una participación activa y dinámica de parte de los estudiantes y, a la vez, le permite al profesor evaluar el grado de comprensión adquirido por los estudiantes al seguir las órdenes (Richards y Rodgers, 2001, p. 76). En sus primeras etapas, los estudiantes sólo reciben órdenes y las ejecutan, sin producir nada oralmente, por ejemplo, el estudiante escucha: ¡Pablo, abra la puerta, por favor! Pablo se levanta y ejecuta la acción. Conforme los estudiantes avanzan en su aprendizaje, las órdenes son más complejas y extensas y, en algunas ocasiones, más adelante en el proceso de enseñanza-aprendizaje, son los mismos estudiantes los que dan órdenes a su profesor o a otros compañeros. De nuevo, la destreza auditiva es un elemento primordial en este método, ya que sin ella los estudiantes no podrían seguir las órdenes o instrucciones del profesor. A pesar de su total aceptación y uso en el ámbito educativo, este método presenta una limitante para los estudiantes, ya que el aprendizaje de un idioma no radica solamente en seguir o dar órdenes o instrucciones. Actualmente, se considera como una técnica muy útil en niveles básicos e intermedios.

Otro método que se desarrolla en los años 60, es el denominado Sugestopedia o Desugestopedia introducido por G. Lozanov. Brown (2000) menciona que "este método pone en práctica el concepto de aprendizaje significativo, basado en una técnica que enfatiza la relajación mental para así obtener la mayor información posible" (p. 105). En esta metodología, la música es un elemento esencial para neutralizar las inhibiciones de los estudiantes en las etapas iniciales de su aprendizaje y eliminar todas aquellas limitaciones falsas impuestas por la cultura. A pesar del énfasis que se da a la traducción de la lengua meta a la lengua materna, se hace uso de textos leídos en voz alta por el profesor, al compás de la música para que los estudiantes, primeramente, sigan la lectura del profesor viendo su texto y, luego, escuchen la lectura sin ver el texto. Como se puede observar, este método no resalta la comprensión auditiva con ejercicios específicos destinados para el desarrollo de ésta, pero se de la introducción de la lectura de textos en voz alta, la cual no se considera una actividad muy dinámica, pero sí efectiva. Pese a su innovador método musical, la Sugestopedia no tuvo mayor auge y no se conoce en la actualidad de libros de texto que promuevan su uso. 
Alrededor de los años 70, aparece el Método Natural con su máximos exponentes S. Krashen y T. Terrell. En este método se enfatiza el contacto con la lengua más que la práctica, lo que prepara emocionalmente al estudiante para el aprendizaje. Asimismo, no se permite el uso de la lengua materna, ya que lo que se pretende es la comunicación oral en la lengua meta. Dentro de un ambiente libre de tensiones y muy motivador, los estudiantes experimentan un período de silencio en sus primeras etapas, durante el cual solo reciben información (input). La producción oral no se da hasta que los estudiantes se sientan listos para hablar. Richards y Rodgers (2001) sugieren que "el elemento auditivo es muy importante en este método, ya que desde el principio los estudiantes reciben información en la lengua meta tales como, anuncios por altavoces, radio, o televisión, conferencias y diálogos" (p. 179). Según Krashen, los estudiantes reciben la información tal y como sucede en la realidad y van construyendo la competencia lingüística necesaria para comunicarse con fluidez (Krashen, 1982, p. 114). Este método puntualiza la necesidad de utilizar materiales auditivos auténticos, como los citados anteriormente por Richards y Rodgers, con el objetivo de preparar al estudiante para enfrentar situaciones cotidianas de una forma más exitosa, natural y espontánea. Muchos de los elementos introducidos por Krashen y Terrell en este método son aún considerados importantes en la enseñanza de una lengua extranjera, tales como: el orden natural o predecible de la adquisición de algunas estructuras gramaticales, la importancia de una atmósfera positiva y la importancia de una auto-corrección constante y apropiada (monitoring) por parte de los estudiantes. Sin embargo, la diferencia tan radical que plantea Krashen entre el aprendizaje consciente de un idioma (learning) y el aprendizaje inconsciente del mismo (acquisition) lo lleva a concluir que una persona nunca podrá adquirir un segundo idioma a través del aprendizaje consciente de éste, ni a utilizarlo inconscientemente como un hablante nativo. Este factor, entre otras razones, desmerece profundamente el método y actualmente no son muchos sus seguidores.

En los años 70 nace también el Método Comunicativo con D. A. Wilkins, como su principal precursor. Este método enfatiza la importancia de la comprensión auditiva para desarrollar una competencia comunicativa en los estudiantes, más allá de un simple conocimiento gramatical. Para este método, la enseñanza de una lengua extranjera debe dar igual importancia a las cuatro macro destrezas (producción oral, comprensión auditiva, producción escrita y comprensión de lectura). Para este método es de gran utilidad el material contextualizado y auténtico que lleve a una negociación espontánea y real del significado en situaciones diarias en la vida de los estudiantes. Dentro de las actividades utilizadas en este método para el desarrollo de la destreza auditiva, se encuentran ejercicios 
como: completar tablas y gráficos; llenar espacios en blanco; contestar preguntas; discriminar información falsa o verdadera; tomar apuntes o hacer resúmenes, entres otras. Por su dinamismo e integración de las cuatro destrezas, este método ha sido ampliamente aceptado y actualmente goza de un numeroso grupo de seguidores.

La década de los 90 es testigo del surgimiento de varios métodos, tales como el Enfoque Basado en el Uso de Estrategias (Strategy-Based Approach); la Instrucción Basada en Contenidos (Content-Based Instruction); el Enfoque por Tareas Específicas (Task-Based Approach) y la Teoría de las Inteligencias Múltiples (Multiple Intelligence Theory). La columna vertebral de estas tendencias es la integración de todas las macro destrezas, el énfasis de los diferentes estilos y estrategias de aprendizaje, y el uso de materiales auténticos y contextualizados con temas de interés para los estudiantes. En el Enfoque Basado en el Uso de Estrategias, la piedra angular es la instrucción cotidiana de la utilización de las estrategias en forma sistematizada para aprender a escuchar. De esta manera, el estudiante toma conciencia de cómo funciona el idioma y de las estrategias que puede utilizar para el aprendizaje del mismo. En la Instrucción Basada en Contenidos y en el Enfoque por Tareas Específicas, una de las actividades que se impulsa es escuchar charlas o conferencias acerca de un tema de interés para los alumnos, quienes toman apuntes, resumen, o completan tablas o gráficos. Luego, se les pide a los estudiantes que investiguen más profundamente sobre el tema y que presenten sus resultados y opiniones en grupos de discusión, debates, o presentaciones individuales. También, se les pide realizar una tarea específica como crear un cuestionario o entrevista, aplicarlo a una población meta, y dar el reporte de los resultados obtenidos. A lo largo de estas actividades, se hace hincapié en los diferentes estilos y estrategias de aprendizaje para que los estudiantes se sientan libres de utilizar sus propias habilidades en forma natural (Richards y Rodgers, 2001, pp. 204-242). En cuanto a la Teoría de las Inteligencias Múltiples se refiere, con sus nueve inteligencias (lingüístico-verbal, espacial-visual, lógico-matemática, musical, corporal, natural, intrapersonal, interpersonal, y existencial) introducidas por H. Gardner, la destreza auditiva va de la mano con las inteligencias lingüístico-verbal e interpersonal, en donde cualquier tipo de intercambio de información requiere de un alto nivel de comprensión auditiva para una comunicación eficaz, y la musical, en la cual los estudiantes ponen atención a cambios de acento, ritmo y entonación (Richards y Rodgers, 2001, pp. 115-124).

Esta breve reseña histórica del rol que ha tenido la destreza auditiva en la enseñanza de una lengua extranjera, permite observar que la misma ha jugado un papel importante en diferentes métodos y, por lo tanto, en el aula a través de ejercicios controlados como la 
repetición y el dictado, y ejercicios menos controlados como la toma de apuntes y el resumen de las ideas más importantes de una charla.

\section{Referencias}

Brown, D.H. (2000). Principles of Language Learning and Teaching. New York: Longman.

Cabrera, L. (1986). Bases para un Diseño de Cursos de Inglés para la Secundaria. Tesis: Universidad Autónoma de México.

Celce-Murcia, M. (1991). Teaching English as a Second of Foreign Language. Second Edition. NY: Newbury House.

Freeman, D. (2000). Techniques and Principles in Language Teaching. Second Edition Oxford University Press.

Gardner, H. (1993). Multiple Intelligences: The theory in practice. New York: Basic Books.

González, L. (1976). Influencia Extranjera en el Desarrollo Científico y Educativo de Costa Rica. San José: Editorial Costa Rica.

González, L. (1978). Evolución de la Instrucción Pública en Costa Rica. San José: Editorial Costa Rica.

Krashen, S. (1982). Principles and Practice in Second Language Acquisition. Oxford: Pergamon Press.

Ministerio de Educación Pública, Costa Rica. (1972). Programa de Inglés para el Primer Ciclo. San José: Publicaciones del MEP.

Monge, C. \& Rivas, F. (1978). La Educación: Fragua de Nuestra Democracia. San José: Editorial Costa Rica.

Quesada, J. (1993). Educación en Costa Rica: 1821-1940. San José: EUNED.

Ramírez, Marlene. (2004). Comunicación personal. Entrevista realizada a la M.Sc. Anabelle Venegas F., Oficina de Lenguas Extranjeras, Ministerio de Educación Pública, realizada el 25 de octubre del 2004.

Richards, J. and Rodgers, T. (2001). Approaches and Methods in Language Teaching. London: Cambridge University Press. 